\title{
Research Article \\ Effect of Hydration on Physicochemical Properties of End-Capped PLGA
}

\author{
Susan D'Souza, ${ }^{1}$ Rossella Dorati, ${ }^{2}$ and Patrick P. DeLuca ${ }^{3}$ \\ ${ }^{1}$ Sunovion Pharmaceuticals Inc., Marlborough, MA 01752, USA \\ ${ }^{2}$ Department of Drug Sciences, Section of Pharmaceutical Chemistry and Technology, University of Pavia, \\ Viale Taramelli 12, 27100 Pavia, Italy \\ ${ }^{3}$ University of Kentucky, College of Pharmacy, Lexington, KY 40536, USA
}

Correspondence should be addressed to Susan D’Souza; dr_ssdsouza@yahoo.com

Received 2 May 2014; Accepted 27 June 2014; Published 13 August 2014

Academic Editor: Fraser Buchanan

Copyright ( 2014 Susan D’Souza et al. This is an open access article distributed under the Creative Commons Attribution License, which permits unrestricted use, distribution, and reproduction in any medium, provided the original work is properly cited.

The objective of this study was to assess the physicochemical effects of hydrating a hydrophobic end-capped poly(lactide-coglycolide) (PLGA) polymer in the liquid and vapor state. PLGA RG503 polymer was incubated at $37^{\circ} \mathrm{C}$ in $0.5 \%$ polyvinyl alcohol (PVA) solution and at $90 \% \mathrm{RH}$. Samples were withdrawn at predetermined intervals and changes to polymer properties like glass transition temperature $\left(\mathrm{T}_{\mathrm{g}}\right)$, moisture uptake, molecular weight change, and $\%$ acid number were determined using differential scanning calorimetry, Karl Fisher titrimetry, gel permeation chromatography, and acid base titrimetry, respectively. Study results showed that $\mathrm{T}_{\mathrm{g}}$ was depressed instantaneously upon hydration, indicating that bulk water acted as a plasticizer of hydrophobic end-capped PLGA. $\mathrm{T}_{\mathrm{g}}$ values decreased to levels below the incubation temperature when hydrated in $0.5 \%$ PVA solution but not in $90 \% \mathrm{RH}$. The drop in $\mathrm{T}_{\mathrm{g}}$ exhibited a linear relationship $\left(R^{2}>0.99\right)$ to the amount of water uptake by the polymer; higher moisture uptake was noted with liquid water. Removal of moisture from the polymer matrix resulted in recovery of $\mathrm{T}_{\mathrm{g}}$, only up to a period of 14 days. Presence of water in liquid or vapor form caused a reduction in molecular weight of the polymer and a corresponding increase in $\%$ acid number over the duration of the study.

\section{Introduction}

Hydrated polymer systems have been widely investigated owing to the effect of water on the performance of commercial polymers and the critical role played by water-polymer interactions in biological processes. In the presence of water, a polymer may exhibit changes in mechanical, chemical, rheological, and transport properties [1]. Depending on the chemical nature of the polymer, the presence of water may result in certain desirable or undesirable changes, causing a profound impact on the behavior of the polymer. Understandably, the manipulation of the rate and extent of moisture in a polymer has been the subject of much research, notably in the field of dosage form design and drug delivery [2-4].

Amongst other properties, amorphous polymers are often characterized by their glass transition temperature $\left(\mathrm{T}_{\mathrm{g}}\right)$ that represents the point at which the solid glassy or highly viscous brittle polymer transitions into a less viscous, more mobile, rubbery state. In its solid glassy state, the polymer is practically immobile. Once in a rubbery state, the polymer chains become more flexible resulting in greater molecular mobility. With higher flexibility and molecular mobility at temperatures above the $\mathrm{T}_{\mathrm{g}}$, there is greater propensity for the polymer to undergo physical and chemical changes. From the literature, it is well established that the presence of water alters the $\mathrm{T}_{\mathrm{g}}$ of polymers [5]. Indeed, water, a small molecule, is known to act as plasticizer; that is, it reduces the $T_{g}$ of amorphous polymers and alters their elastic modulus [6]. In other instances, water has been shown to form stable bridges between polymer chains through hydrogen bonding (antiplasticizer) [7].

Thus, any natural and synthetic polymer that possesses hydrophilic groups like hydroxyl, carboxyl, and carbonyl groups can be expected to have strong and/or weak interactions with water. Through this interaction, physical properties such as thermal properties of the polymer and water are 
markedly influenced. With most polymers, water is expected to act as a potent plasticizer, similar to its behavior with a wide range of amorphous and partially amorphous pharmaceutical solids [8]. It appears to act in the same way as other low molecular weight plasticizers.

Several amorphous pharmaceuticals exist in the glassy state under ambient conditions [9]. The adsorption of water to these solid pharmaceuticals is known to cause plasticization, resulting in a concentration-dependent lowering of $\mathrm{T}_{\mathrm{g}}[5,10]$. Water incursion, leading to adsorption, can occur during both the manufacture of the pharmaceutical dosage form and storage, potentially resulting in profound changes in the physicochemical properties of the amorphous phase [11]. It is no surprise that residual water contained in the dosage form after manufacturing remains an important consideration in dosage form development. This parameter could be of considerable practical significance, particularly in view of the amorphous nature of the pharmaceutical solid with the concomitant possibility of plasticization effects [12]. Additionally, the effects of moisture on stability of pharmaceutical formulations cannot be overlooked.

The effects of hydration on chemical, mechanical, and transport properties of traditional polymers like modified celluloses (HPMC, HPC) are well documented by pharmaceutical researchers $[2,13]$. Changes in moisture uptake of the polymer, leading to swelling and its eventual dissolution, have been exploited in several conventional dosage forms like controlled release tablets. As such, polymer dissolution in aqueous media involves absorption or adsorption of water at easily accessible regions on the polymer, breakdown of polymer-polymer bonds, generation of water-polymer bonds, loosening of polymer chains, swelling, and finally dissolution of polymer chains in an aqueous medium.

For polymers that are biodegradable, the effects of water bear additional significance. Studies have shown that physicomechanical changes in mechanical properties, glass transition temperature, polymer degradation, and so forth, of dry and wet biodegradable polymer films were strongly affected by the presence of water in the matrix [14]. Though research on biodegradable polymers used as carriers for therapeutics has certainly peaked, little is known about the stability of the biodegradable polymers and their formulations while in solid state.

Of the many biodegradable polymers available for use, poly(lactide-co-glycolides) (PLGA) have been the subject of considerable research for a number of reasons. In addition to their biodegradability, they are biocompatible, nontoxic, and nonimmunogenic and have been approved for human use by the US Food and Drug Administration [15]. Dosage forms prepared using PLGA polymers are extremely popular as they offer the possibility of sustained release of a wide range of therapeutics, including peptides and proteins $[16,17]$.

The ability of the PLGA polymer to prolong drug release stems from its chemical and physical nature. As a polyester based polymer, PLGA is available in ratios that contain an ester bond between the slow degrading lactide and the fast degrading glycolide in random fashion throughout the polymer chain. Thus, lactide:glycolide ratios of $85: 15,65: 35$, $50: 50$, and so forth are fairly common and frequently

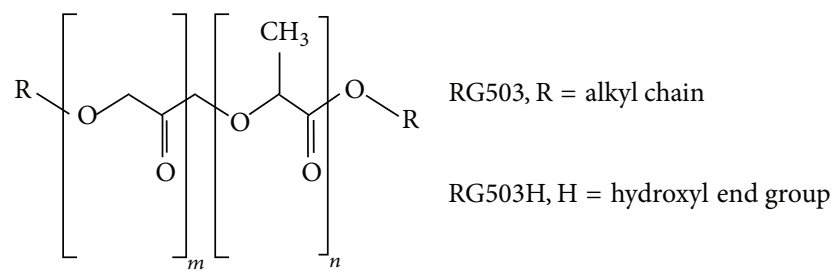

FIgURE 1: Chemical structure of poly(D,L-lactide-co-glycolide) (PLGA).

reported in the literature [18-22]. Altering the copolymer composition (lactide : glycolide ratio) is a primary means to tailor drug levels in vivo for varying duration, from weeks to several months [23-25], leading to an improvement in patient compliance due to reduced dosing frequency [19, 26-28]. Another property of the PLGA polymer that has been used to customize drug release is its molecular weight [29-31]. Intuitively, it has been established that the larger molecular weight PLGAs degrade slower than the lower molecular weight polymer. Despite numerous publications discussing the effect of copolymer composition and molecular weight on drug delivery, little is known about the effect of endcapping of the PLGA polymer. End-capping of the PLGA polymer refers to the presence of an alkyl ester as the terminal group in the polymer (Figure 1) instead of the commonly observed acidic functionalities, that is, lactide and glycolide species, in the polymer, by a methyl or some similar chemical moieties. In the literature, end-capped PLGAs are typically referred to as hydrophobic PLGAs (e.g., RG503) and are easily distinguished from their more hydrophilic counterparts (e.g., $\mathrm{RG} 503 \mathrm{H}$ ) by the absence of an " $\mathrm{H}$ " at the end of the polymer descriptor.

Thus, the goal of this research was to evaluate the effects of hydrating a hydrophobic poly(lactide-co-glycolide) polymer in the liquid and vapor state and identify parameters critical to polymer degradation. Various experiments were performed to identify and elucidate the changes that occur upon moisture ingress and their impact on polymer behavior and discuss its impact on designing dosage forms containing hydrophobic biodegradable polymers.

\section{Materials and Methods}

2.1. Materials. Hydrophobic poly(lactide-co-glycolide) (PLGA, Mw = $38 \mathrm{kDa}$ ), RG503 (50:50 lactide : glycolide), was obtained from Boehringer Ingelheim, Ingelheim, Germany. Polyvinyl alcohol (PVA) $(\mathrm{Mw}=30,000-70,000 \mathrm{Da})$ was purchased from Sigma (Aldrich Chemical Company, USA) and $\mathrm{KNO}_{3}$ from Fisher Scientific (USA). All the solvents used were of analytical grade.

\subsection{Methods}

2.2.1. Hydration of Hydrophobic PLGA in Liquid and Vapor State. Approximately $50 \mathrm{mg}$ of dried polymer was incubated in deionized water containing $0.5 \%$ PVA and stored at $37 \pm$ $2^{\circ} \mathrm{C}$ in an electronically controlled oven. At predetermined 
intervals, samples were removed, filtered, rinsed with deionized water, and dried under vacuum until constant weight. Similarly, a second set of samples were weighed and placed in open vials over saturated salt solution $\left(\mathrm{KNO}_{3}\right), 90 \% \mathrm{RH}$, at $37 \pm 2^{\circ} \mathrm{C}$. At predetermined intervals, samples were removed and vacuum-dried until constant weight. Finally, a set of control samples were exposed to dry heat in an oven at $37 \pm 2^{\circ} \mathrm{C}$.

2.2.2. Determination of $T_{g}$. Glass transition temperature $\left(T_{g}\right)$ was determined using a modulated differential scanning calorimetry (MDSC) on 2929 Modulated DSC (TA Instruments, USA) equipped with a refrigerated cooling system (RCS). Samples of about 5-10 mg were weighed into aluminum pans that were hermetically sealed and subjected to two cooling and heating cycles from $-60^{\circ} \mathrm{C}$ to $60^{\circ} \mathrm{C}$ at cooling and heating rates of $5^{\circ} \mathrm{C} / \mathrm{min}$, for a period of 40 seconds and an amplitude of $\pm 0.5^{\circ} \mathrm{C}$. The DSC cell and RCS were purged with dry nitrogen at $60 \mathrm{~mL} / \mathrm{min}$ and $120 \mathrm{~mL} / \mathrm{min}$, respectively. The system was calibrated using an Indium standard. Data were treated with Thermal Solutions software (TA Instruments, UK) and the results expressed as the mean of three determinations.

2.2.3. Molecular Weight Determination. The molecular weight of polymer was determined by Gel Permeation Chromatography (GPC). The GPC system consisted of two Ultrastyragel columns connected in series $(7.8 \times 300 \mathrm{~mm}$ each, one with $10^{4} \AA$ pores and one with $10^{3} \AA$ pores), a pump (Shimadzu LC-6A, Japan), UV detector set at $\lambda=210 \mathrm{~nm}$ (Shimadzu, Japan), and software to compute molecular weight distribution (Waters, Maxima 820, Milford, USA). Sample solutions in tetrahydrofuran (THF) at a concentration of $5 \mathrm{mg} / \mathrm{mL}$ were filtered through a $0.45 \mu \mathrm{m}$ filter (Millipore, USA) before injection into the GPC system and were eluted with THF at $0.4 \mathrm{~mL} / \mathrm{min}$. The weight-average molecular weight $\left(M_{w}\right)$ of each sample was calculated using monodisperse polystyrene standards, $M_{w} 1,000-50,000 \mathrm{Da}$.

2.2.4. Water Content Determination by Karl Fisher Titration. The water uptake in samples was measured by a Brinkmann $684 \mathrm{KF}$ Coulometer Metrohm. At predetermined intervals, $1 \mathrm{~mL}$ of anhydrous methanol was added to the samples prepared as described in Section 2.2.1, and the samples were occasionally shaken for 1 hour. A small volume $(\mu \mathrm{L})$ of methanol was withdrawn and injected into the titration vessel. The volume of sample used was based on the anticipated water content and desired degree of accuracy. Each measurement was performed in triplicate and the water content $(w / w)$ was calculated using the following equation:

$$
\text { Water content }(\%)=100 \times \frac{\left(M_{\text {water }}-M_{\text {blank }}\right)}{M_{\text {initial }}} \text {, }
$$

where $M_{\text {water }}$ is the mass of water in the sample, $M_{\text {blank }}$ is the mass of water in methanol, and $M_{\text {initial }}$ is the initial mass of the polymer.

2.2.5. Acid Number Determination. Acid number (AN), a measure of the acid content in the polymer, is directly related

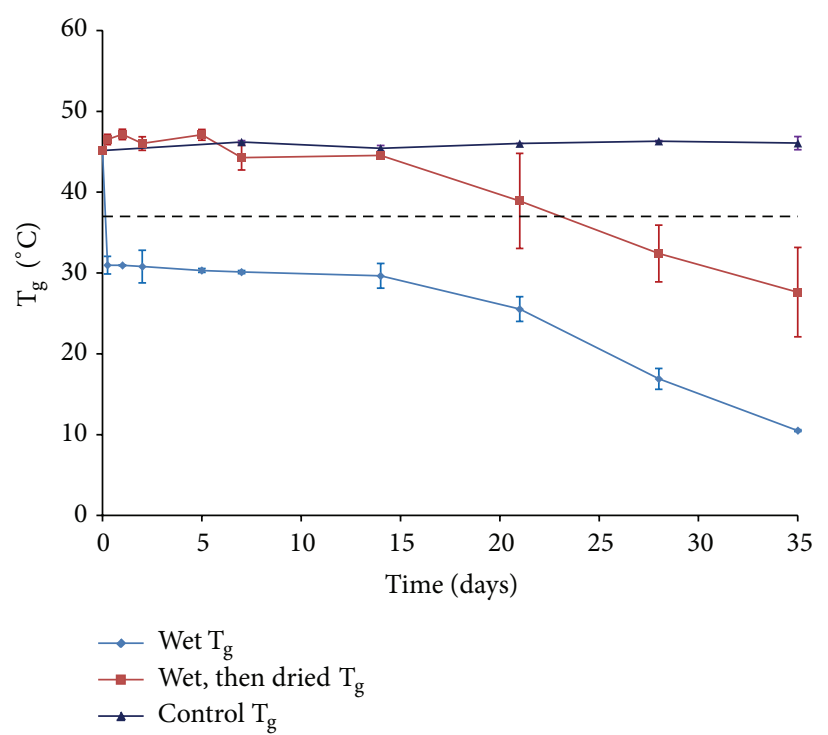

FIGURE 2: Glass transition temperature of RG503 after incubation in $0.5 \%$ PVA solution at $37^{\circ} \mathrm{C}$ and after drying. Dotted line represents incubation temperature $\left(37^{\circ} \mathrm{C}\right)$.

to the number of free carboxylic acid functionalities and was determined by titration as previously described [32]. Briefly, polymer samples were dissolved in $20-40 \mathrm{~mL}$ of a mixture of acetone/tetrahydrofuran $(1: 1)$ and immediately titrated with $0.01 \mathrm{~N}$ methanolic potassium hydroxide $(\mathrm{KOH})$ to a stable pink end point using phenolphthalein as an indicator. Each measurement was performed in triplicate:

$$
\mathrm{AN}=\mathrm{mL}_{\text {sample }} \times \mathrm{N}_{\mathrm{KOH}} \times \frac{\mathrm{MW}_{\mathrm{KOH}}}{\text { Weight of PLGA }(\mathrm{gm})} .
$$

The $\%$ acid number was obtained by normalizing the acid number with the total acid number of the polymer. The total acid number was determined by incubating polymer samples at $55^{\circ} \mathrm{C}$ up to complete degradation.

\section{Results and Discussion}

3.1. Effect of Hydration on Glass Transition Temperature $\left(T_{g}\right)$. The effect of water in solution form (0.5\% PVA) on the glass transition temperature $\left(\mathrm{T}_{\mathrm{g}}\right)$ of hydrophobic PLGA RG503 is illustrated in Figure 2. From an initial value of $45.15^{\circ} \mathrm{C}$, a sudden drop in $\mathrm{T}_{\mathrm{g}}\left(\sim 14^{\circ} \mathrm{C}\right)$ was observed within a mere 6 hours of exposure to water (Wet $\mathrm{T}_{\mathrm{g}}$ ). In fact, the value at the 6-hour time point was below the incubation temperature of $37^{\circ} \mathrm{C}$ (dotted line, Figure 2). Given that the PLGA used in this study was hydrophobic and end-capped, the sharp initial decrease in glass transition temperature was rather surprising. After the initial drop in $\mathrm{T}_{\mathrm{g}}$, values appeared to plateau between 29 and $31^{\circ} \mathrm{C}$ through 14 days after which a steady descent in values was noted through the last time point (day 35). Thus, the drop in $\mathrm{T}_{\mathrm{g}}$ appeared to be triphasic; that is, the first phase was characterized by an initial drop, followed by a plateau, and finally the third phase where a steady 


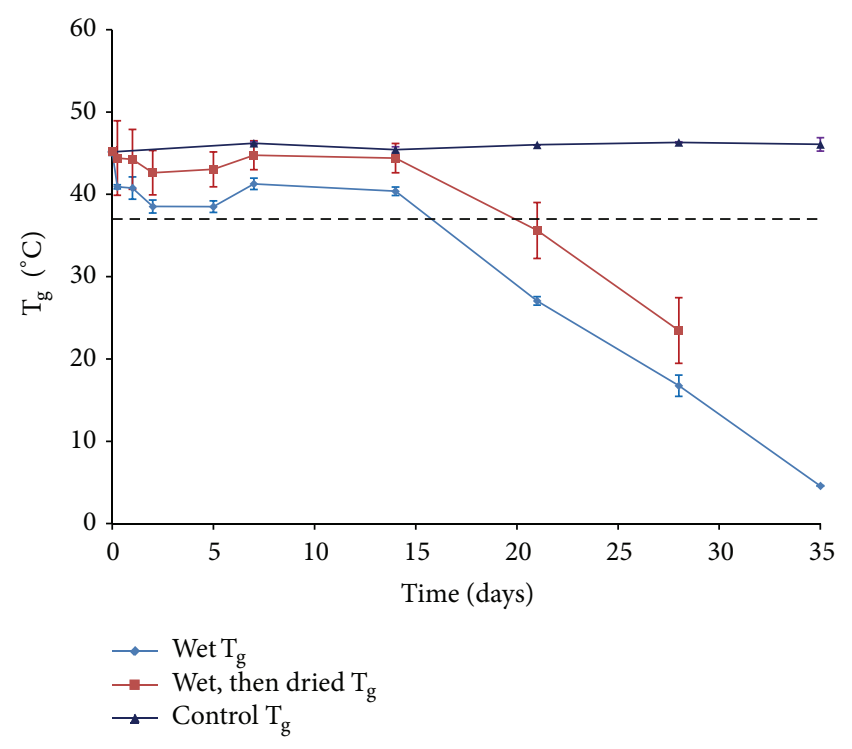

FIGURE 3: Glass transition temperature of RG503 after incubation in $90 \% \mathrm{RH}$ at $37^{\circ} \mathrm{C}$ and after drying. Dotted line represents incubation temperature $\left(37^{\circ} \mathrm{C}\right)$.

decrease in $\mathrm{T}_{\mathrm{g}}$ values was observed to reach approximately $10.5^{\circ} \mathrm{C}$ by day 35 .

A similar triphasic behavior was used to describe the $T_{g}$ of the wet polymer after drying. For nearly 14 days, the dried polymer samples exhibited nearly constant $\mathrm{T}_{\mathrm{g}}$ values ranging between 45 and $47^{\circ} \mathrm{C}$; that is, the polymer appeared to regain its initial $\mathrm{T}_{\mathrm{g}}$ value after drying. Thus, once dried, the polymer maintained its $\mathrm{T}_{\mathrm{g}}$ above the incubation temperature of $37^{\circ} \mathrm{C}$. After the 2-week time point, the polymer no longer appeared to recover its $\mathrm{T}_{\mathrm{g}}$. This phase was manifested by a consistent drop in polymer $\mathrm{T}_{\mathrm{g}}$ after drying and continued through the last measurement ( 35 days). Interestingly, the rate of drop in the $\mathrm{T}_{\mathrm{g}}$ in the dried polymer samples was similar to that of the samples incubated in $0.5 \%$ PVA solution.

Figure 2 also describes a third set of samples (control) that was exposed to dry heat $\left(37^{\circ} \mathrm{C}\right.$, no moisture). Unlike the polymer exposed to $0.5 \%$ PVA solution, the control samples did not exhibit any increase or decrease in $\mathrm{T}_{\mathrm{g}}$ during the entire course of the experiment; rather, the $\mathrm{T}_{\mathrm{g}}$ was almost constant, with values above the incubation temperature of $37^{\circ} \mathrm{C}$ indicating that, in the absence of moisture, storage of the polymer at a temperature below its $\mathrm{T}_{\mathrm{g}}$ did not alter its glass transition temperature.

Figure 3 illustrates the effect of water in vapor form $(90 \%$ $\mathrm{RH})$ on the glass transition temperature $\left(\mathrm{T}_{\mathrm{g}}\right)$ of hydrophobic PLGA RG503. In a manner similar to Figure 2, the profile obtained with the incubated polymer $\left(\mathrm{Wet}_{\mathrm{g}}\right.$ ) was triphasic, albeit to a lower degree in the first two phases. During the first phase, the $\mathrm{T}_{\mathrm{g}}$ dropped to $\sim 41^{\circ} \mathrm{C}$ at the 6-hour time point (initial value $=45.15^{\circ} \mathrm{C}$ ). This drop in $\mathrm{T}_{\mathrm{g}}$ was much less than what was observed at the 6-hour time point with the samples incubated in $0.5 \%$ PVA solution. Additionally, the 6-hour hydrated $\mathrm{T}_{\mathrm{g}}$ value for the $90 \% \mathrm{RH}$ polymer sample remained noticeably above the incubation temperature of $37^{\circ} \mathrm{C}$ (dotted line, Figure 3), in clear contrast to the value recorded in Figure 2 with the $0.5 \%$ PVA sample. Once hydrated, the $T_{g}$ values for the $90 \% \mathrm{RH}$ samples remained nearly constant through 14 days $\left(38-41^{\circ} \mathrm{C}\right)$ and above the incubation temperature. However, there was a drastic drop in hydrated $\mathrm{T}_{\mathrm{g}}$ values after the 2-week time point, the rate of which was much faster than that noted with the corresponding samples in Figure 2. By day 35 , the incubated $\mathrm{T}_{\mathrm{g}}$ value had dropped to $4.5^{\circ} \mathrm{C}$.

Similar to the results obtained with the polymer incubated in 0.5\% PVA solution and dried (Figure 2), samples incubated at $90 \% \mathrm{RH}$ and dried (Figure 3) appeared to recover their $\mathrm{T}_{\mathrm{g}}$ values through a period of 14 days. These values were very close to the $\mathrm{T}_{\mathrm{g}}$ of the raw polymer $\left(45.15^{\circ} \mathrm{C}\right)$. After day 14, the $\mathrm{T}_{\mathrm{g}}$ of the incubated and dried samples dropped sharply at a rate nearly identical to that observed with the incubated polymer $\mathrm{T}_{\mathrm{g}}$ (Figure 3 ) to reach a value of $23^{\circ} \mathrm{C}$ at day $28 . \mathrm{T}_{\mathrm{g}}$ of the incubated and dried sample at day 35 could not be determined.

3.2. Moisture Uptake. From the literature, PLGA polymers are known to be biodegradable thermoplastic materials and as such are amorphous and adsorb water but do not dissolve in aqueous solutions [6]. Water ingress leads to series of notable events that eventually lead to degradation of the PLGA polymer. Initial incursion of water leads to polymer hydration, after which degradation ensues, and finally solubilization of the polymer [1,3-5]. Hence, measurement of moisture uptake by the polymer during the initial stages of hydration is crucial to the understanding of the biodegradation process.

From Figures 2 and 3, it is clear that exposure to water caused a drop in $\mathrm{T}_{\mathrm{g}}$ of the end-capped PLGA (RG503). Using Karl Fisher titration as described in Section 2.2.4, moisture content upon initial hydration of the RG503 polymer was measured after 6 hours, 1,2 , and 5 days by incubating polymer samples at $37^{\circ} \mathrm{C}$ in both $0.5 \%$ PVA solution (liquid water) and $90 \% \mathrm{RH}$ (water vapor). Figure 4 shows the results of the moisture measurements. From an initial moisture content of $0.026 \%$, exposure to either $0.5 \%$ PVA solution or $90 \% \mathrm{RH}$ resulted in the RG503 polymer imbibing additional amounts of water. Interestingly, the amount of water uptake by the polymer incubated in $0.5 \%$ PVA was severalfold greater than that observed under $90 \% \mathrm{RH}$, presumably due to the presence of a greater quantity of liquid bulk water that was available to penetrate the polymer to a much higher extent.

Regardless of whether the water was present in liquid or vapor state, a few trends in water uptake were noted.

(a) Exposure to water caused a spike in moisture levels (initial hydration) in the hydrophobic end-capped RG503 polymer at the 6 -hour time point.

(b) The phase of water (liquid versus vapor) determined the rate and extent of initial hydration.

(c) After initial hydration, the rate of moisture uptake was reasonably steady and nearly linear through day 5 .

(d) By day 5, water uptake by the polymer incubated at $0.5 \%$ PVA solution and $90 \%$ RH was $20 \%$ and $2 \%$, respectively. 


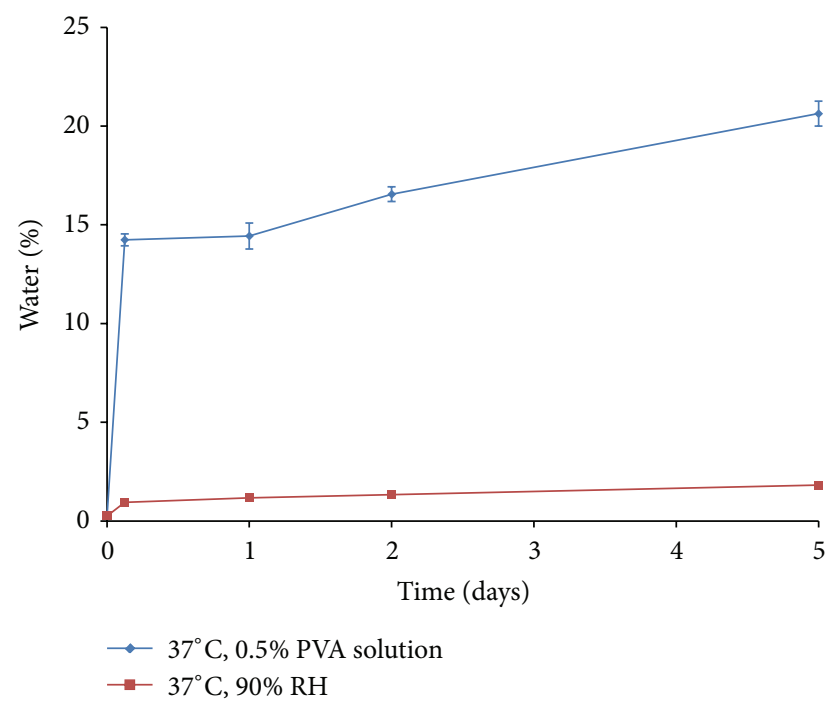

Figure 4: Water uptake in PLGA polymer RG503 after incubation at $37^{\circ} \mathrm{C}$ in $0.5 \%$ PVA solution and $90 \% \mathrm{RH}$ through 5 days.

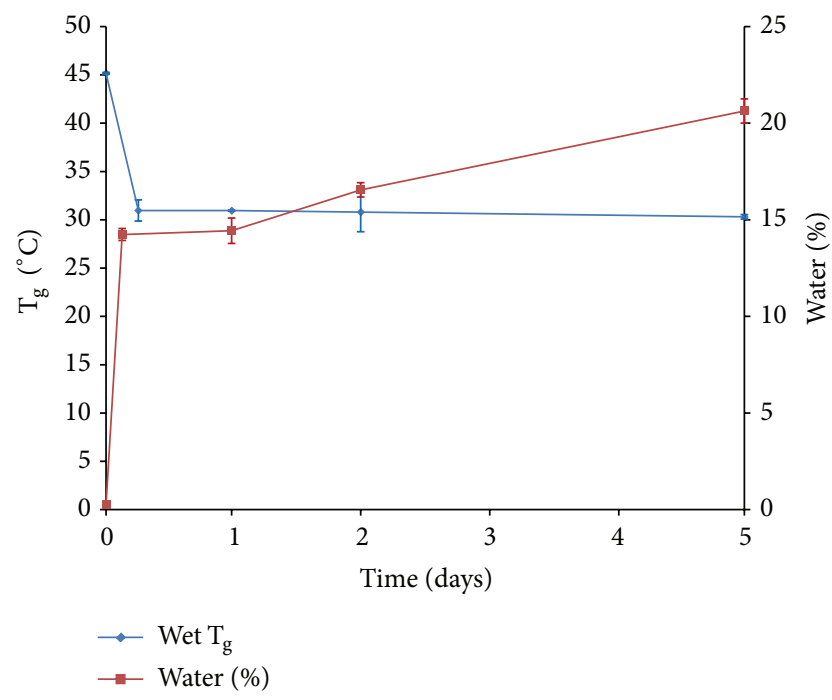

FIGURE 5: Comparison of glass transition temperature $\left(\mathrm{T}_{\mathrm{g}}\right)$ of PLGA polymer RG503 and water content of samples incubated at $37^{\circ} \mathrm{C}$ in $0.5 \%$ PVA solution through day 5.

3.3. Correlation between Moisture Uptake and $T_{g}$. From the data in Figure 5, there is a sharp increase in water content of the RG503 polymer at $37^{\circ} \mathrm{C}$ in $0.5 \%$ PVA solution (6-hour time point) and a steep drop in glass transition temperature to below the incubation temperature, $37^{\circ} \mathrm{C}$. After that, the $\mathrm{T}_{\mathrm{g}}$ remains virtually unchanged $\left(\sim 30^{\circ} \mathrm{C}\right)$ through 5 days while the moisture content rises modestly from 14 to $20 \%$. The data in Figure 5 indicate that water incursion into the polymer impacts chain mobility; that is, the polymer has transitioned from a glassy rigid state to a rubbery, more flexible state and is manifested by a drop in $\mathrm{T}_{\mathrm{g}}$. These findings are in excellent agreement with similar reports on other polymers

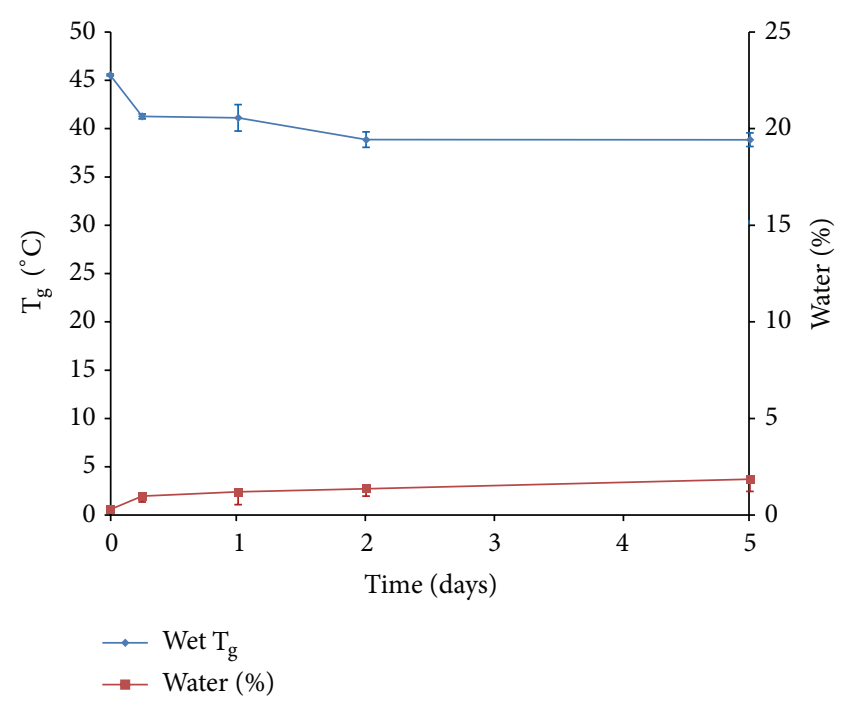

FIGURE 6: Comparison of glass transition temperature $\left(\mathrm{T}_{\mathrm{g}}\right)$ of PLGA polymer RG503 and water content of samples incubated at $37^{\circ} \mathrm{C}$ in $90 \%$ RH through day 5 .

like polyvinylpyrrolidone (PVP), modified celluloses, and so forth $[33,34]$.

Similar findings are observed in Figure 6, where a drop in glass transition temperature, albeit small, is noted after 6 hours of incubation in $90 \% \mathrm{RH}$ at $37^{\circ} \mathrm{C}$ and corresponds to a slight uptake in water content in the RG503 hydrophobic endcapped polymer. After the 6-hour time point, the $\mathrm{T}_{\mathrm{g}}$ is nearly unchanged, that is, slow decrease from $41^{\circ} \mathrm{C}$ at 6 hours to $38^{\circ} \mathrm{C}$ at 5 days, paralleling the observations noted with polymer water uptake ( $1 \%$ at 6 hours to $2 \%$ at 5 days). Surprisingly, the water uptake data with the RG503 polymer at day 5 were nearly similar to previous findings reported by our group on the more hydrophilic RG503H polymer [6]. While the results in Figure 6 describe similarity in water uptake, the drop in glass transition temperature with the end-capped polymer was significantly lower than the hydrophilic $503 \mathrm{H}$ polymer [6].

The results of the moisture uptake and $\mathrm{T}_{\mathrm{g}}$ experiments can be correlated by understanding the effect of water on the polymer. As mentioned earlier, water, a small molecule, having a glass transition temperature of around $-135^{\circ} \mathrm{C}$, exerts a plasticizing effect on the RG503 polymer $[5,35]$. Upon incubation in $0.5 \%$ PVA solution or $90 \% \mathrm{RH}$ at $37^{\circ} \mathrm{C}$, the glass transition temperature of the polymer was rapidly depressed to levels below the incubation temperature. However, the decrease in $\mathrm{T}_{\mathrm{g}}$ was reversible, at least through a period of 14 days. Interestingly, regardless of the physical state of water used, the relationship between moisture uptake and glass transition temperature in the initial hydration period was linear (Figure 7) and an excellent correlation $\left(R^{2}>\right.$ 0.99) was obtained for both sets of samples, that is, polymer incubated in $0.5 \%$ PVA and $90 \% \mathrm{RH}$. Additionally, the value of the intercept ( $0 \%$ moisture) for $90 \% \mathrm{RH}$ data set was $46.75^{\circ} \mathrm{C}$, close to the $\mathrm{T}_{\mathrm{g}}$ value $\left(45.15^{\circ} \mathrm{C}\right)$ of the raw RG503 endcapped polymer. 


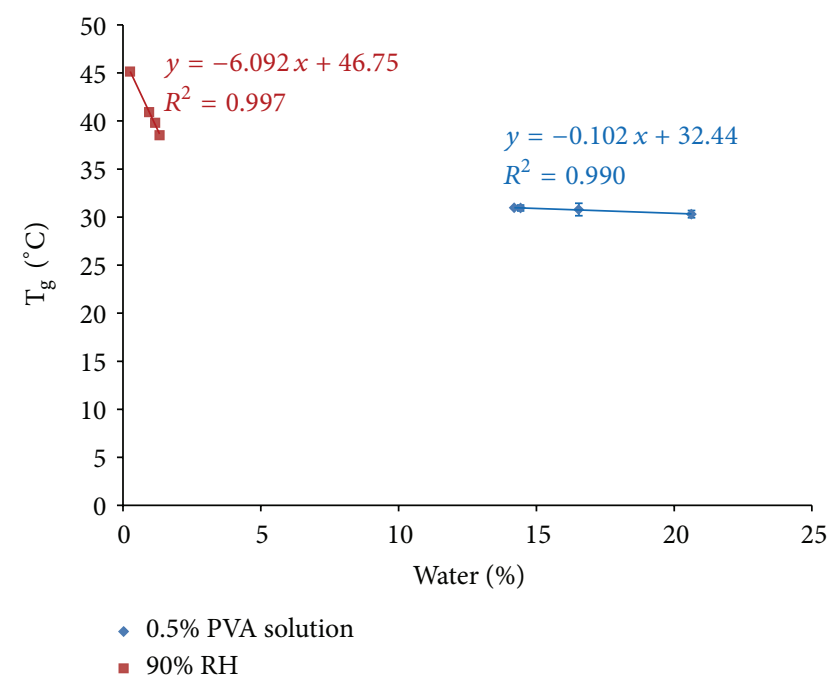

FIGURE 7: Relationship between glass transition temperature and moisture content for PLGA polymer RG503.

Figure 6 also reveals that the drop in $T_{g}$ is extremely profound with incremental increases in water uptake that were observed upon incubation in $90 \%$ RH. Further uptake of water as noted with samples exposed to $0.5 \%$ PVA solution did not impact the $\mathrm{T}_{\mathrm{g}}$ significantly. This aspect is clearly evident in slopes presented in Figure 7; that is, the slope for $\mathrm{T}_{\mathrm{g}}$ decrease with $90 \%$ RH was early sixty-fold greater than that seen with $0.5 \%$ PVA solution. The difference in slopes can be explained by the fact that though direct incubation in $0.5 \%$ PVA solution led to imbibing of greater quantities of water, most of it could not diffuse into the hydrophobic polymer matrix and was probably associated with the surface. In fact, the literature reports that discuss the interaction of water with hydrophilic polymers have shown that the thermodynamic properties of absorbed water are altered relative to those of bulk water [36, 37]. Using spectroscopic and calorimetric techniques, moisture present in hydrophilic polymer has been previously categorized as

(a) bulk (free) water that is freezable (Type I) and not strongly associated with the polymer,

(b) bound water that is freezable (Type II) as it is not as closely associated with the polymer,

(c) bound water that is not freezable (Type III) as it is closely associated with the polymer matrix [7, 37, 38].

From the results of the current study, it is apparent that a significant portion of the water present in the polymer (incubated at $0.5 \%$ PVA solution) was not readily available to exert its plasticizing effect and exhibited behavior that is characteristic of bulk water.

3.4. Effect of Hydration on Molecular Weight Change. The effect of water on the degradation of the end-capped RG503 polymer is shown in Figure 8. Of particular interest is the fact that polymer degradation profiles were independent of the state of the water molecules employed in the incubation study.

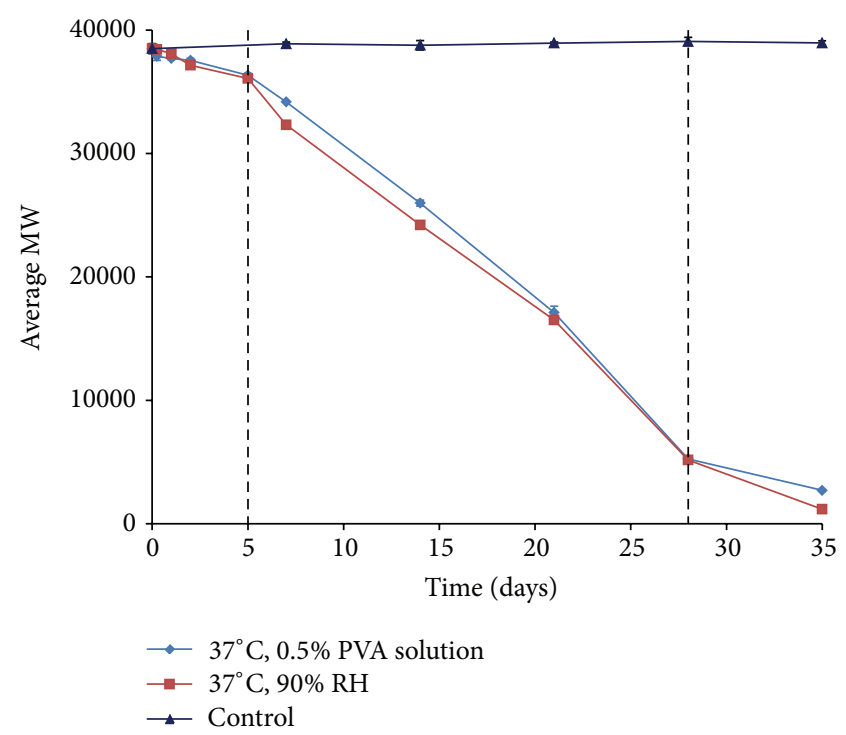

Figure 8: Time-dependent molecular weight changes in PLGA polymer RG503 incubated in $0.5 \%$ PVA solution, $90 \% \mathrm{RH}$, and dry heat at $37^{\circ} \mathrm{C}$ (control).

Similar to the $\mathrm{T}_{\mathrm{g}}$ curves, molecular weight decrease exhibited a triphasic profile. Region 1 ( $0-5$ days) was characterized by a slow degradation rate and Region 2 (5-28 days) displayed a high rate of molecular weight reduction, while a slowing of polymer molecular weight described Region 3 (28 days onwards). Indeed, the $\%$ drop in molecular weight was a mere $6 \%$ in Region 1, rising to reach $86 \%$ in Region 2, after which the $\%$ drop was muted in Region 3 though greater than Region 1 . Hence, the $\%$ drop exhibited a rank order where $\%$ drop in Region $2>$ Region $3>$ Region 1.

The molecular weight decrease exhibited by the endcapped RG503 PLGA polymer can be explained by the critical role of water in the degradation process. For most polymers, water absorption is the first step that occurs when a polymeric sample is incubated in an aqueous environment [39]. In Figure 8, days 0-5 (Region 1) describe the molecular weight changes during the initial stages of hydration. Water diffuses through the polymer chains, loosening the rigid glassy structure and increasing flexibility [40]. From Figures 2 and 3, polymer $\mathrm{T}_{\mathrm{g}}$ dropped sharply due to hydration and subsequently recovered after drying. Indeed, the $\mathrm{T}_{\mathrm{g}}$ recovery of the polymer could be explained by the fact that molecular weight dropped very slightly ( 6\%) in Region 1 (Figure 8).

However, when considering biodegradable polyester polymers like PLGA, water molecules not only act as a plasticizer, but also initiate hydrolysis of the ester bonds in the polymer chain. Hydrolytic degradation increases polymer hydrophilicity, that is, increase in the number of carboxylic acid end groups. As such, presence of acid end groups has a dual effect on the hydrophobic end-capped PLGA polymer in that it increases the water uptake ability of the polymer and initiates autocatalytic effects in the polymer [23]. Unlike some other polymers, PLGAs undergo a bulk polymer degradation process; that is, the rate of penetration of water (hydration) is much faster than solubilization of the polymer [41]. Thus, the 


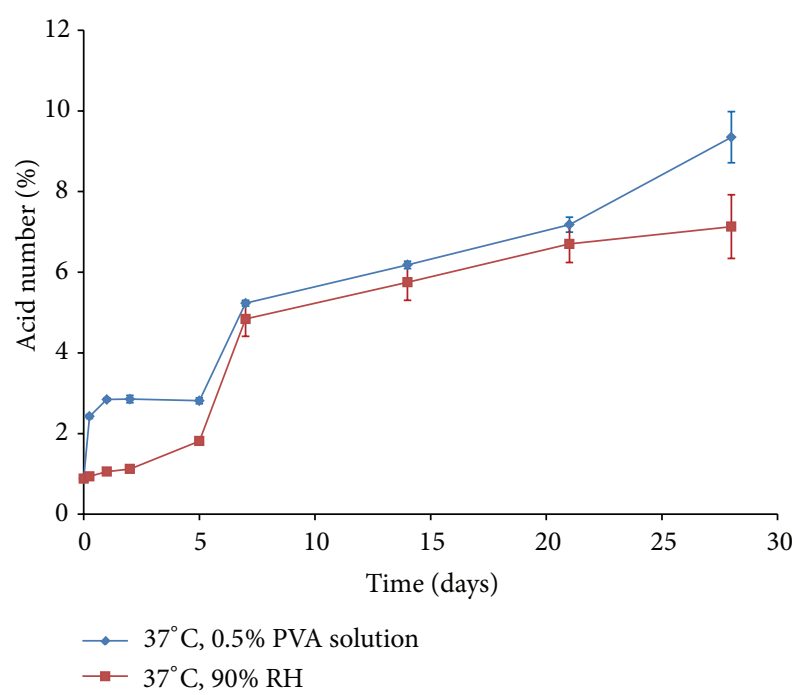

Figure 9: Changes in the terminal carboxyl group content in PLGA polymer RG503 incubated in $0.5 \%$ PVA solution or $90 \% \mathrm{RH}$ at $37^{\circ} \mathrm{C}$.

eventual outcome of hydration is ester bond hydrolysis that causes cleavage of the polymer backbone leading to a drop in molecular weight which is clearly described by Region 2 (Figure 8). In Region 3, polymer degradation rate slowed down slightly due to the fact that the hydrolysis reaction had proceeded to reach a point where very little of the polymer matrix was remaining.

3.5. Effect of Hydration on Acid Number. Figure 9 describes the effect of hydration on the $\%$ acid number of hydrophobic PLGA RG503. For the samples stored in 0.5\% PVA solution, incursion of water causes an approximately 2.5 -fold increase in the $\%$ acid number from the initial time point to 6 hours. Subsequently, the \% acid number appears to plateau until day 5 after which there occurs a steady rise through day 28 . For the samples incubated in $90 \% \mathrm{RH}$, the profile at the early time points is slightly different. In the vapor state, the $\%$ acid number rises gradually through day 5 after which a sudden spike in values is observed at day 7 , and then, a steady increase through day 28.

When comparing the two profiles in Figure 9, it is clear that from the 6 -hour time point through day 5 , the $\%$ acid number generated in samples incubated in $0.5 \%$ PVA solution is significantly greater than those hydrated using $90 \% \mathrm{RH}$. At day 7 , the $\%$ acid number values are nearly similar and appear to be independent of the mode of hydration, and this trend continues through day 21 . Finally, the values appear to slightly diverge once again (day 28) and are higher for the samples incubated in $0.5 \%$ PVA solution.

In a manner similar to molecular weight changes (Figure 8 ), the $\%$ acid number is a reflection of the hydrolytic degradation that occurs inside the hydrophobic PLGA polymer. For samples hydrated using 0.5\% PVA solution, the amount of water uptake was significantly higher at the early time points (Figure 4), ensuring rapid hydrolysis of ester bonds and an increase in the acidic functionalities in the
PLGA matrix (Figure 9). Subsequently, constant attack by water molecules led to an increase in \% acid number over time for the bulk degrading PLGA. In contrast, the water uptake in samples incubated in $90 \% \mathrm{RH}$ was significantly lower (Figure 5) and explains the low \% acid number values at the early time points (Figure 9). However, the carboxylic acid end groups formed in the samples incubated at $90 \% \mathrm{RH}$ are unable to diffuse out of the polymer, causing an acidic microenvironment inside the matrix leading to autocatalysis. The extensive polymer chain hydrolysis increases polymer hydrophilicity, that is, increase in the number of acidic moieties and eventually polymer breakdown $[42,43]$.

\section{Conclusions}

Exposure of a hydrophobic end-capped PLGA polymer to water in liquid and vapor state caused a drop in glass transition temperature $\left(\mathrm{T}_{\mathrm{g}}\right)$ and polymer plasticization, the extent depending on the mode of hydration. An excellent correlation was observed between the drop in $\mathrm{T}_{g}$ and the amount of water uptake by the polymer when hydrated in liquid and vapor states. Presence of water also resulted in polymer hydrolysis, decrease in molecular weight, and an increase in acid number. Results from the current study suggest that when hydrated, hydrophobic end-capped PLGA polymers undergo several physical and chemical changes that can impact biological performance of drug delivery systems that use these polymers as to encapsulate therapeutic agents.

\section{Conflict of Interests}

The authors declare that there is no conflict of interests regarding the publication of this paper.

\section{Acknowledgments}

The research described in this paper was performed while the authors were affiliated with the University of Kentucky, Lexington, KY. The authors wish to thank Oakwood Labs, Oakwood, $\mathrm{OH}$, and the Graduate School, University of Kentucky, Lexington, KY, for their financial support.

\section{References}

[1] H. N. Joshi and T. D. Wilson, "Calorimetric studies of dissolution of hydroxypropyl methylcellulose E5 (HPMC E5) in water," Journal of Pharmaceutical Sciences, vol. 82, no. 10, pp. 1033-1038, 1993.

[2] J. Siepmann and N. A. Peppas, "Modeling of drug release from delivery systems based on hydroxypropyl methylcellulose (HPMC)," Advanced Drug Delivery Reviews, vol. 48, no. 2-3, pp. 139-157, 2001.

[3] J. Grund, M. Koerber, M. Walther, and R. Bodmeier, "The effect of polymer properties on direct compression and drug release from water-insoluble controlled release matrix tablets," International Journal of Pharmaceutics, vol. 469, pp. 94-101, 2014.

[4] B. Saša, P. Odon, S. Stane, and K. Julijana, "Analysis of surface properties of cellulose ethers and drug release from their matrix 
tablets," European Journal of Pharmaceutical Sciences, vol. 27, no. 4, pp. 375-383, 2006.

[5] B. C. Hancock and G. Zografi, "The relationship between the glass transition temperature and the water content of amorphous pharmaceutical solids," Pharmaceutical Research, vol. 11, no. 4, pp. 471-477, 1994.

[6] P. Blasi, S. S. D’Souza, F. Selmin, and P. P. DeLuca, "Plasticizing effect of water on poly(lactide-co-glycolide)," Journal of Controlled Release, vol. 108, no. 1, pp. 1-9, 2005.

[7] H. Hatakeyama and T. Hatakeyama, "Interaction between water and hydrophilic polymers," Thermochimica Acta, vol. 308, no. 12, pp. 3-22, 1998.

[8] N. E. Hunter, C. S. Frampton, D. Q. M. Craig, and P. S. Belton, "The use of dynamic vapour sorption methods for the characterisation of water uptake in amorphous trehalose," Carbohydrate Research, vol. 345, no. 13, pp. 1938-1944, 2010.

[9] J. Kerč and S. Srčič, “Thermal analysis of glassy pharmaceuticals," Thermochimica Acta, vol. 248, pp. 81-95, 1995.

[10] M. Gordon and J. S. Taylor, "Ideal copolymers and the secondorder transitions of synthetic rubbers. i. non-crystalline copolymers," Journal of Applied Chemistry, vol. 2, pp. 493-500, 1952.

[11] R. Surana, L. Randall, A. Pyne, N. M. Vemuri, and R. Suryanarayanan, "Determination of glass transition temperature and in Situ study of the plasticizing effect of water by inverse gas chromatography," Pharmaceutical Research, vol. 20, no. 10, pp. 1647-1654, 2003.

[12] N. Passerini and D. Q. M. Craig, "An investigation into the effects of residual water on the glass transition temperature of polylactide microspheres using modulated temperature DSC," Journal of Controlled Release, vol. 73, no. 1, pp. 111-115, 2001.

[13] C. B. McCrystal, J. L. Ford, and A. R. Rajabi-Siahboomi, "A study on the interaction of water and cellulose ethers using differential scanning calorimetry," Thermochimica Acta, vol. 294, no. 1, pp. 91-98, 1997.

[14] H. Kranz, N. Ubrich, P. Maincent, and R. Bodmeier, "Physicomechanical properties of biodegradable poly(D, L-lactide) and poly(D, L-lactide-co-glycolide) films in the dry and wet states," Journal of Pharmaceutical Sciences, vol. 89, no. 12, pp. 1558-1566, 2000.

[15] S. D’Souza, J. A. Faraj, S. Giovagnoli, and P. P. DeLuca, "Preparation, characterization and in vivo evaluation of Olanzapine Poly(D, L-lactide-co-glycolide) (PLGA) microspheres," Journal of Pharmaceutics, vol. 2013, Article ID 831381, 9 pages, 2013.

[16] E. Comets, F. Mentré, F. Nimmerfall et al., "Nonparametric analysis of the absorption profile of octreotide in rabbits from long-acting release formulation OncoLAR," Journal of Controlled Release, vol. 59, no. 2, pp. 197-205, 1999.

[17] Y. Capan, B. H. Woo, S. Gebrekidan, S. Ahmed, and P. P. DeLuca, "Preparation and characterization of poly (D, L-Lactide-CoGlycolide) microspheres for controlled release of Poly(LLysine) complexed plasmid DNA," Pharmaceutical Research, vol. 16, pp. 509-513, 1999.

[18] E. J. Park, D. H. Na, and K. C. Lee, "In vitro release study of mono-PEGylated growth hormone-releasing peptide-6 from PLGA microspheres," International Journal of Pharmaceutics, vol. 343, pp. 281-283, 2007.

[19] J. Xuan, Y. Lin, J. Huang et al., "Exenatide-loaded PLGA microspheres with improved glycemic control: in vitro bioactivity and in vivo pharmacokinetic profiles after subcutaneous administration to SD rats," Peptides, vol. 46, pp. 172-179, 2013.
[20] L. Sun, S. Zhou, W. Wang, X. Li, J. Wang, and J. Weng, "Preparation and characterization of porous biodegradable microspheres used for controlled protein delivery," Colloids and Surfaces A: Physicochemical and Engineering Aspects, vol. 345, pp. 173-181, 2009.

[21] V. Karagkiozaki, E. Vavoulidis, P. G. Karagiannidis et al., “Development of a nanoporous and multilayer drug-delivery platform for medical implants," International Journal of Nanomedicine, vol. 7, pp. 5327-5328, 2012.

[22] N. Zheng, M. Zhou, C. Du, S. Wang, and W. Lu, "5-fluorouracil delivery from a novel three-dimensional micro-device: in vitro and in vivo evaluation," Archives of Pharmacal Research, vol. 36, no. 12, pp. 1487-1493, 2013.

[23] S. D'Souza, J. A. Faraj, S. Giovagnoli, and P. P. DeLuca, "Development of risperidone PLGA microspheres," Journal of Drug Delivery, vol. 2014, Article ID 620464, 11 pages, 2014.

[24] S. Yandrapu and U. B. Kompella, "Development of sustainedrelease microspheres for the delivery of SAR 1118, an LFA-1 antagonist intended for the treatment of vascular complications of the eye," Journal of Ocular Pharmacology and Therapeutics, vol. 29, pp. 236-248, 2013.

[25] V. Nandakumar, V. Geetha, S. Chittaranjan, and M. Doble, "High glycolic poly (DL lactic co glycolic acid) nanoparticles for controlled release of meropenem," Biomedicine \& Pharmacotherapy, vol. 67, pp. 431-436, 2013.

[26] S. D’Souza, J. A. Faraj, and P. P. DeLuca, "Microsphere delivery of Risperidone as an alternative to combination therapy," European Journal of Pharmaceutics and Biopharmaceutics, vol. 85, pp. 631-639, 2013.

[27] R. B. Shmueli, M. Ohnaka, A. Miki et al., "Long-term suppression of ocular neovascularization by intraocular injection of biodegradable polymeric particles containing a serpin-derived peptide," Biomaterials, vol. 34, no. 30, pp. 7544-7551, 2013.

[28] H. H. Kwak, W. S. Shim, M. K. Son et al., "Efficacy of a new sustained-release microsphere formulation of exenatide, DA3091, in Zucker diabetic fatty (ZDF) rats," European Journal of Pharmaceutical Sciences, vol. 40, no. 2, pp. 103-109, 2010.

[29] S. D'Souza, J. A. Faraj, S. Giovagnoli, and P. P. DeLuca, "IVIVC from long acting olanzapine microspheres," International Journal of Biomaterials, vol. 2014, Article ID 407065, 11 pages, 2014.

[30] J. Braunecker, M. Baba, G. E. Milroy, and R. E. Cameron, “The effects of molecular weight and porosity on the degradation and drug release from polyglycolide," International Journal of Pharmaceutics, vol. 282, pp. 19-34, 2004.

[31] B. Li, J. Yang, L. Ma, F. Li, Z. Tu, and C. Gao, "Influence of the molecular weight of poly(lactide-co-glycolide) on the in vivo cartilage repair by a construct of poly(lactideco-glycolide)/fibrin gel/mesenchymal stem cells/transforming growth factor- $\beta 1$," Tissue Engineering A, vol. 20, pp. 1-11, 2014.

[32] J. Schrier and P. DeLuca, "Recombinant human bone morphogenetic protein-2 binding and incorporation in PLGA microsphere delivery systems," Pharmaceutical Development and Technology, vol. 4, pp. 611-621, 1999.

[33] J. Teng, S. Bates, D. A. Engers, K. Leach, P. Schields, and Y. Yang, "Effect of water vapor sorption on local structure of poly(vinylpyrrolidone)," Journal of Pharmaceutical Sciences, vol. 99, pp. 3815-3825, 2010.

[34] A. M. Agrawal, R. V. Manek, W. M. Kolling, and S. H. Neau, "Studies on the interaction of water with ethylcellulose: effect of polymer particle size," AAPS PharmSciTech, vol. 4, pp. 469-479, 2003. 
[35] T. Chen, A. Fowler, and M. Toner, "Literature review: supplemented phase diagram of the trehalose-water binary mixture," Cryobiology, vol. 40, no. 3, pp. 277-282, 2000.

[36] M. Yasushi, L. Makoto, and K. Hiromi, "Vibrational spectroscopic study on the structure of water in polymer systems," Journal of Molecular Liquids, vol. 80, no. 2-3, pp. 149-163, 1999.

[37] T. Hatakeyama, K. Nakamura, and H. Hatakeyama, "Vaporization of bound water associated with cellulose fibres," Thermochimica Acta, vol. 352-353, pp. 233-239, 2000.

[38] M. S. Jhon and J. D. Andrade, "Water and hydrogels," Journal of Biomedical Materials Research, vol. 7, no. 6, pp. 509-522, 1973.

[39] S. Li and S. McCarthy, "Further investigations on the hydrolytic degradation of poly (DL-lactide)," Biomaterials, vol. 20, no. 1, pp. 35-44, 1999.

[40] R. M. Ginde and R. K. Gupta, "In vitro chemical degradation of poly(glycolic acid) pellets and fibers," Journal of Applied Polymer Science, vol. 33, no. 7, pp. 2411-2429, 1987.

[41] M. Vert, J. Mauduit, and S. Li, "Biodegradation of PLA/GA polymers: increasing complexity," Biomaterials, vol. 15, no. 15, pp. 1209-1213, 1994.

[42] A. M. Reed and D. K. Gilding, "Biodegradable polymers for use in surgery-poly(glycolic)/poly(Iactic acid) homo and copolymers: 2 . In vitro degradation," Polymer, vol. 22, no. 4, pp. 494-498, 1981.

[43] K. Fu, D. W. Pack, A. M. Klibanov, and R. Langer, "Visual evidence of acidic environment within degrading poly(lactic-coglycolic acid) (PLGA) microspheres," Pharmaceutical Research, vol. 17, no. 1, pp. 100-106, 2000. 

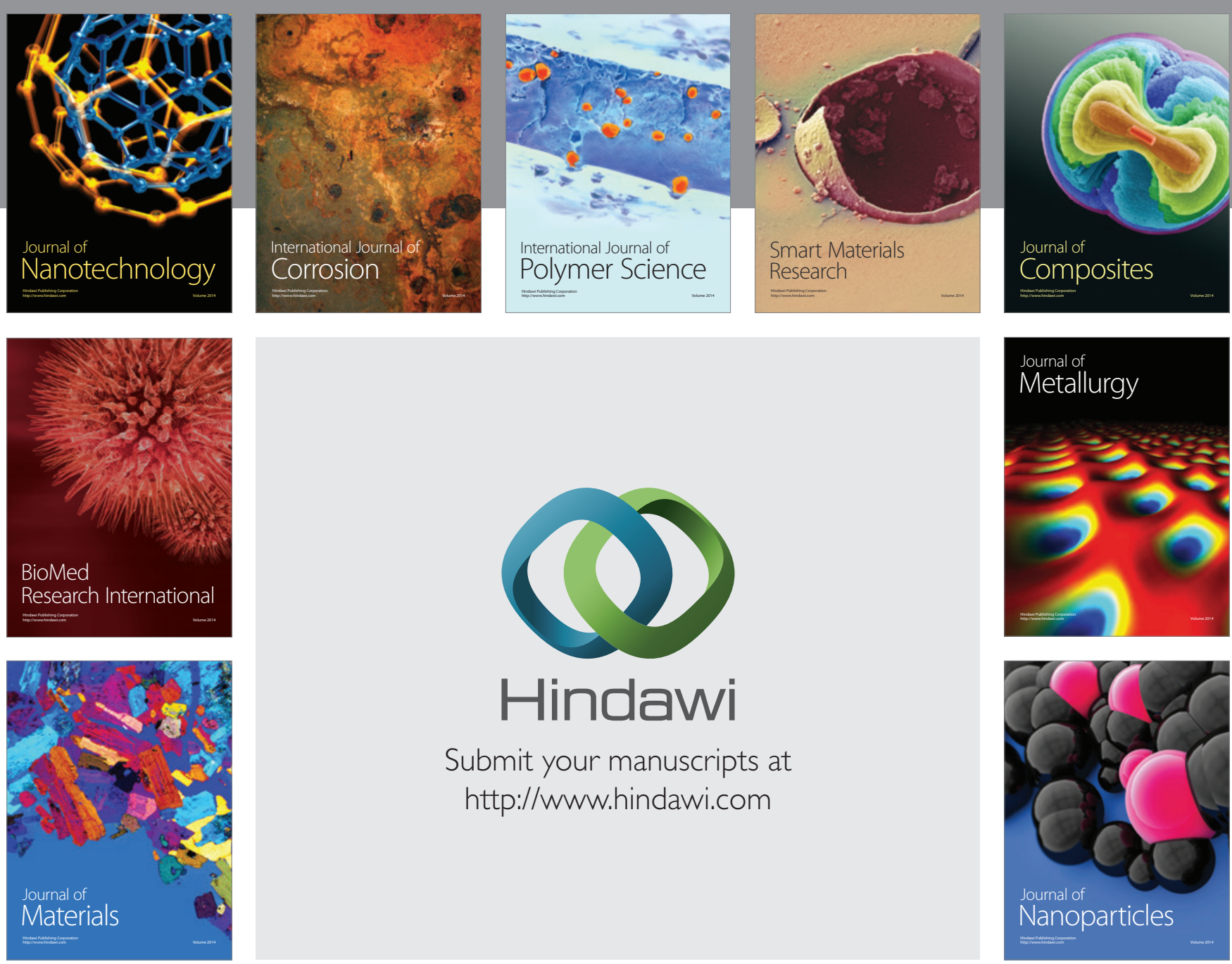

Submit your manuscripts at http://www.hindawi.com
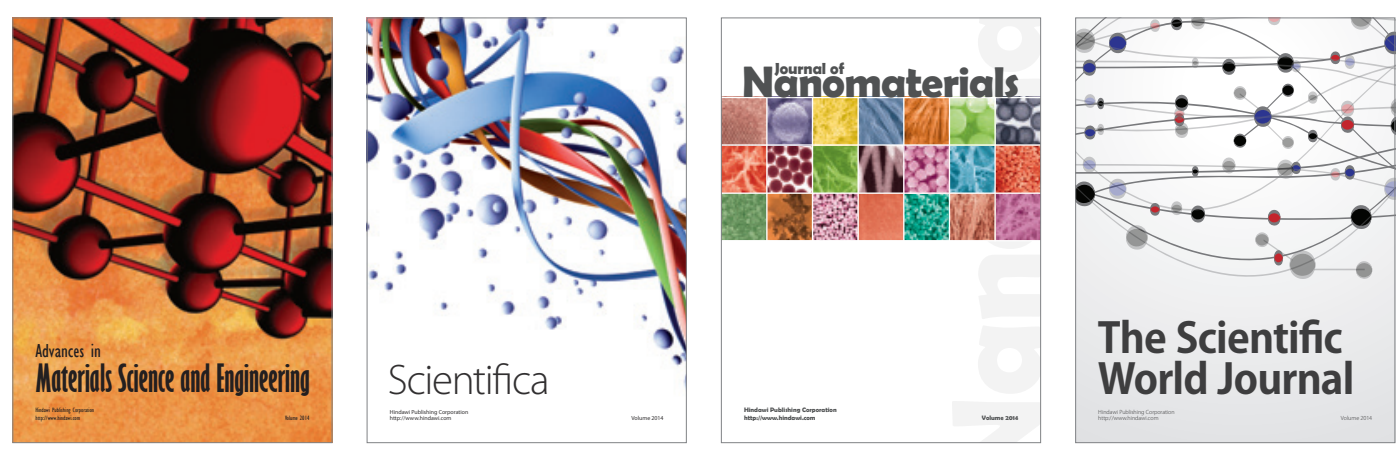

\section{The Scientific World Journal}
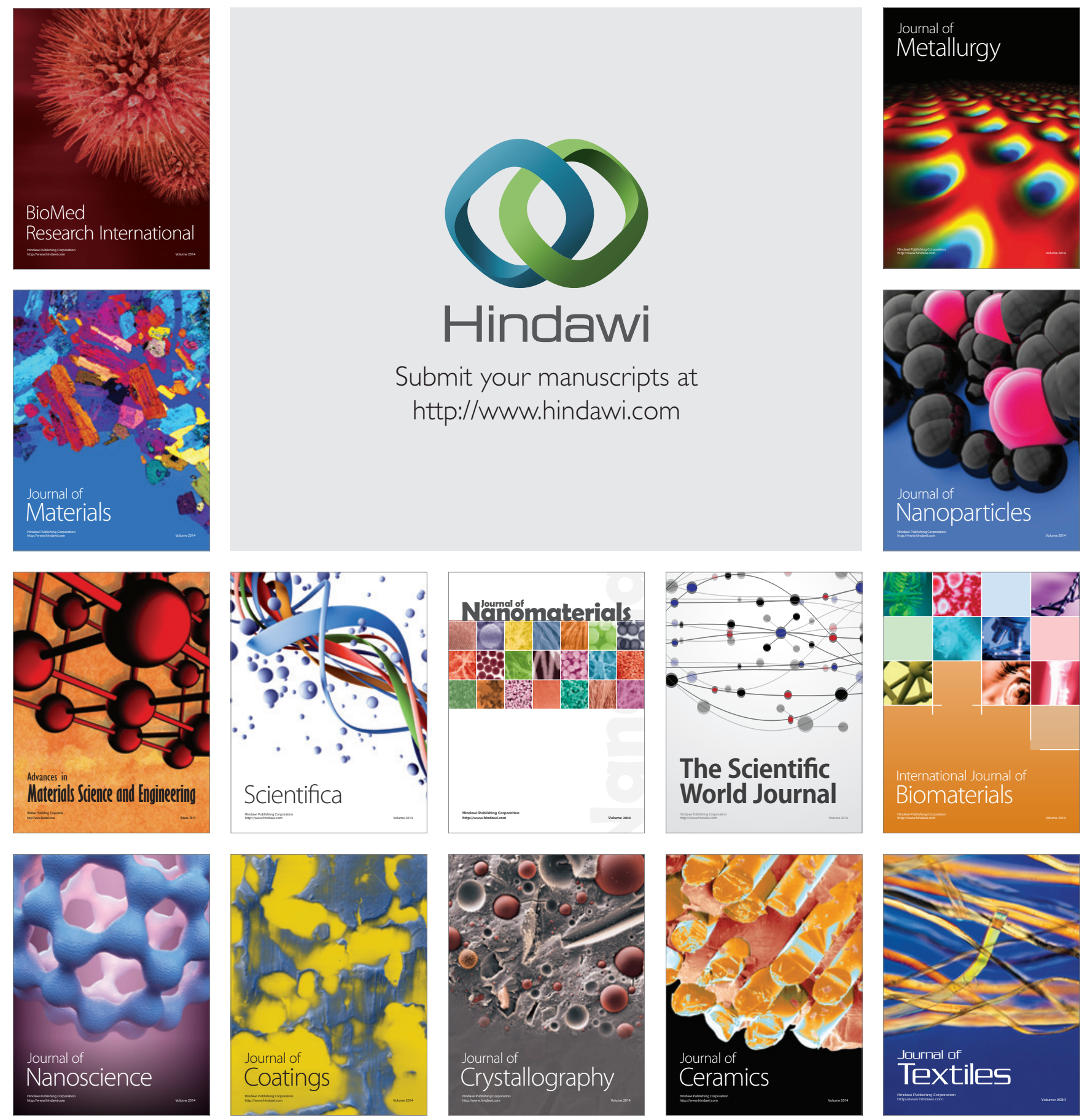